\title{
Submicroscale Flow Sensor Employing Suspended Hot Film with Carbon Nanotube Fins"
}

\author{
Yohei ITO $^{* *}$, Taku HIGUCHI ${ }^{* *}$ and Koji TAKAHASHI ${ }^{* *}$ \\ ${ }^{* *}$ Department of Aeronautics and Astronautics, Kyushu University, \\ 744, Motooka, Nishi-ku, Fukuoka, 819-0395, Japan \\ E-mail: takahashi@aero.kyushu-u.ac.jp
}

\begin{abstract}
A submicroscale flow sensor has been developed that consists of a suspended hot film and carbon nanotube (CNT) fins. Flow measurement experiments, together with a theoretical model, revealed the advantages of the use of CNT fins. The suspended metal film reduces heat loss and the CNT fins enhance the heat transfer to the fluid flow. Herein, the working principle of the CNT fins is presented in detail, together with a description of the micro electro mechanical systems (MEMS) / nano electro mechanical systems (NEMS) techniques used to fabricate the sensor. The CNTs were deposited by a manipulation method that is based on dielectrophoresis.
\end{abstract}

Key words: Submicroscale Flow Sensor, CNT Fin, MEMS, NEMS, Dielectrophoresis

\section{Introduction}

The miniaturization of flow sensors has been the focus of extensive study over the past twenty years, and MEMS technology has contributed significantly to this field. Flow sensors with dimensions on the order of $100 \mu \mathrm{m}$ are usually based on the simple and reliable microthermotransfer principle, where a MEMS membrane or bridging structure provides thermal insulation ${ }^{(1)-(5)}$. Such MEMS thermal flow sensors are now commercially available at low cost due to their superior compatibility with mass-production techniques. However, there is still a continuing demand for ever-higher sensitivity and lower power requirements from smaller sensing elements. Thus, the next goal is further miniaturization for improved microfluidic systems such as those represented by micro total analysis systems $(\mu \mathrm{TAS}){ }^{(6),(7)}$. The current sensor design methods are effective for dimensions of 10 $\mu \mathrm{m}$ and larger, but more attention has to be paid to the development of submicrometer flow sensors, which would even be applicable in the narrow branches of a lab-on-chip.

Basically, a reduction in system size causes degradation of the thermal signal. For example, current MEMS flow sensors use a silicon nitride membrane for thermal insulation ${ }^{(8)}$. This membrane is usually hundreds of nanometers thick, which is sufficient to degrade the thermal signal on a submicrometer scale. This is because the amount of heat transferred from the sensing element to the fluid can be lower than that transferred to the membrane itself. Consequently, the resolution and response of the microthermal sensor are limited by the membrane itself.

The authors have been working on the development of suspended platinum nanofilm sensors, which are sufficiently sensitive to measure the thermal properties of nanomaterials ${ }^{(9)}$. Here, because the thin resistor film on a silicon substrate can be considered to be at the same temperature as the substrate, only the suspended part functions as a heater and sensor. Thus far, a submicroscale fluidic sensor with three platinum hot films (Fig. 1) has been 


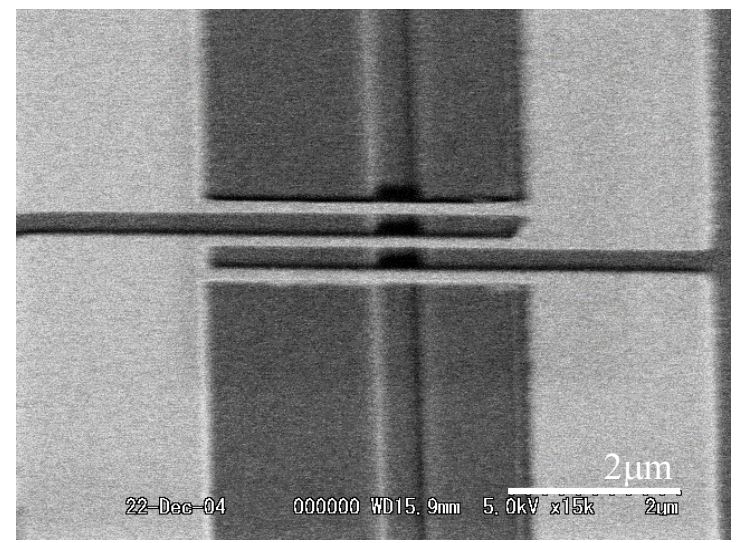

Fig. 1 SEM micrograph of a submicroscale fluidic sensor with three platinum hot films. The platinum hot film is detached from substrate by trench. The suspended length is $500 \mathrm{~nm}$. The width of platinum hot films is $200 \mathrm{~nm}$ and the interval is $800 \mathrm{~nm}$.

tested, where no membrane/bridge structure is used. Each film over a trench is $200 \mathrm{~nm}$ wide, $500 \mathrm{~nm}$ long, and placed at intervals of $800 \mathrm{~nm}$. Gas flow measurements have shown that the conventional sensing principle of convection-induced temperature distribution does not function at this scale. This is because the mean free path of gas flow is comparable to the length scale of the nanosensor and the temperature distribution around the heater tends to be isotropic even in a flow. However, hot-film-based thermal anemometry using a single suspended resistor has been confirmed to work, though the signal from this miniaturized sensor is very small. To improve this submicroscale flow sensor, the current article reports a method for signal enhancement by using carbon nanotube (CNT) fins.

CNTs have attracted a large amount of attention for both electrical and mechanical applications due to their singular properties ${ }^{(10)-(13)}$. The extremely high thermal conductivity of over $2000 \mathrm{~W} \mathrm{~m}^{-1} \mathrm{~K}^{-1}$ and high aspect ratio of over 1000 are considered huge advantages for thermal devices. So far, a new microfluidic sensor employing the hot-wire anemometry principle has been developed using CNTs that are laterally aligned into a $360-\mu \mathrm{m}$-long and $90-\mu \mathrm{m}$-wide conductive trace between two microelectrodes ${ }^{(14)}$. And by extending this technique, a fluidic sensor that is $100 \mathrm{~nm}$ long can be realized. However, this type of configuration results in very low sensitivity of due to the heat diffusion problem mentioned above. The only feasible method to overcome this critical heat loss would involve suspending the CNTs between the two electrodes; however, the resulting sensitivity is not known and such fabrication techniques should increase cost.

The present concept employs a suspended metal film to reduce the heat loss and CNT fins to enhance the heat transfer to the fluid flow. Few other materials besides CNTs would be suitable for such a design: CNTs are not only narrow and have high aspect ratios but are also well-explored as regards synthesis and manipulation for microdevice applications, which makes them particularly suitable for low-cost mass production. Herein we discuss the working principle and fabrication technique of the new hot-film sensor with CNT fins together with experimental results confirming the advantages of the design.

\section{Principle of CNT fin}

A fin provides an extended surface that protrudes from a heated wall, thus enhancing the rate of heat transfer. The increased heat flux causes a larger temperature change for the hot-film, depending on the flow velocity, and thus results in a larger signal from the fluidic sensor. First, we theoretically treat a single CNT fin in an air flow. From the one-dimensional heat conduction model, the heat transfer rate from a fin of uniform cross section $A$ and length $L$ is as follows ${ }^{(15)}$ : 


$$
q_{f}=\sqrt{h P k A} \cdot \theta \frac{\sinh m L+(h / m k) \cosh m L}{\cosh m L+(h / m k) \sinh m L}
$$

Where $m=\sqrt{h P / k A}$ and $h, P, k$, and $\theta$ are the heat transfer coefficient of the fluid, fin perimeter, thermal conductivity of the fin, and temperature difference between the fin base and fluid, respectively. We used CNTs with an average diameter $D$ is $40 \mathrm{~nm}$ and average length of $1 \mu \mathrm{m}$. The thermal conductivity of CNTs is still a controversial topic, but is expected to be $100-1000 \mathrm{~W} \mathrm{~m}^{-1} \mathrm{~K}^{-1}{ }^{(9)}$. On the heat transfer coefficient, the reported experimental values for carbon fiber can be applied as a first assumption; this value is $10-1000 \mathrm{~W} \mathrm{~m}^{-2} \mathrm{~K}^{-1}{ }^{(16)}$. And because of this high thermal conductivity and small cross section, $h / m k<<1.0$ and $m L<<1.0$, which allows for the following approximation:

$$
q_{f}=\sqrt{h P k A} \cdot \theta m L=h \pi D L \theta
$$

This equation implies that fin performance is independent of thermal conductivity. Next consider the number of fins that can be placed on the sensor surface. Here, the fin base fraction $c$ is introduced. If the surface area covered by the fins bases is $c A_{s}(0<c<1)$ and the number of fins $N_{f}$ is $4 c A_{s} / \pi D^{2}$, then the total heat transfer rate is

$$
q_{f-\text { total }}=4 c A_{s} h L \theta / D
$$

This means that the high aspect ratio $L / D$ of a CNT should work to enhance the convective heat transfer of the hot film. Fin performance is estimated in relation to the heat flux without fins, which can be expressed as $q_{s}=A_{s} h^{\prime} \theta$, where $h^{\prime}$ is the heat transfer coefficient for Pt film. In this paper we assess fin performance using the factor $f$ defined as,

$$
f=\frac{q_{f-\text { total }}+(1-c) q_{s}}{q_{s}}=1+c\left(4 \frac{L}{D}-1\right)
$$

where it is assumed that the heat transfer coefficient of a CNT $h$ and that of a Pt film $h$ ' are equal. Thus, the product of a CNT fin's aspect ratio and the fin base fraction $c$ determines the factor $f$. For example, even a small number density of CNT fins can enhance the sensor signal if the fin aspect ratio is very large. This is the most important reason for the use of CNT fins.

\section{Experimental}

\subsection{Fabrication of hot-film sensor}

To build our submicroscale flow sensor, the following MEMS/NEMS techniques were employed: electron beam lithography, the lift-off method for platinum film pattering, isentropic etching of the underside of the sensor.

The sensor element is a suspended Pt nanofilm that is $40 \mathrm{~nm}$ thick, $800 \mathrm{~nm}$ wide, and 6 $\mu \mathrm{m}$ long, but the working principle is that same as that for hot-wire anemometry in conventional flow measurement.

1. A silicon wafer with a 180-nm-thick thermally oxidized layer is used as the starting substrate. This is spin-coated with a 320 -nm-thick electron beam (EB) resist.

2. Sensor resistors and leads are directly drawn on an EB lithography system set up on a Hitachi S-4300 scanning electron microscope (SEM). 
3. Titanium $(5 \mathrm{~nm})$ and platinum $(40 \mathrm{~nm})$ are deposited by physical vapor deposition. The titanium is used only for adhesion.

4. The lift-off method using an EB-resist remover is employed for platinum/titanium film patterning. Only sensor resistors and leads remain on the substrate.

5. The silicon dioxide layer is isotropically etched away using buffered hydrofluoric acid. In this process, both the titanium and dioxide layers beneath the narrow heater/sensor part of platinum film are etched away.

6. Silicon bulk etching is performed with a $\mathrm{KOH}$ solution for $2 \mathrm{~min}$. An etch depth of about $2 \mu \mathrm{m}$ guarantees a completely suspended heater/sensor.

The fabrication process is shown in Fig.2. Here we chose EB lithography because it allows quick changes to the sensor design if necessary. But a scanning stepper is also applicable for this 200-nm sensor, which will guarantee mass production at low cost. Figure 3 presents an SEM micrograph of the sensor, where two sensing elements are set face-to-face with a $100-\mu \mathrm{m}$ interval. This was done to allow a comparison of the sensing performance when CNT fins were added to one of them. Here, both films have a V-shape and not a linear configuration because of the CNT alignment, which is explained later. The inset confirms complete removal of the material underneath this sensor for thermal insulation.

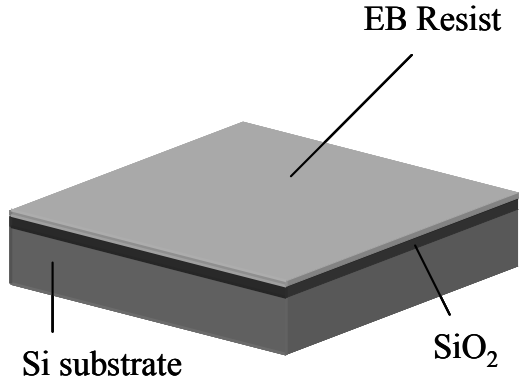

1. Starting material

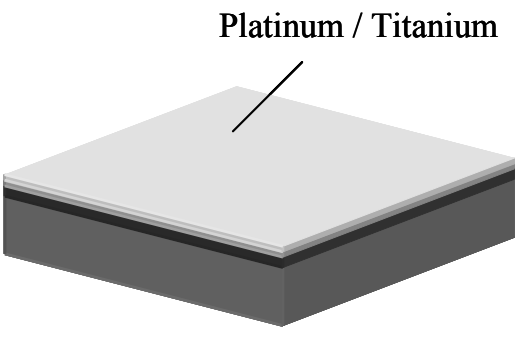

3. Physical vapor deposition

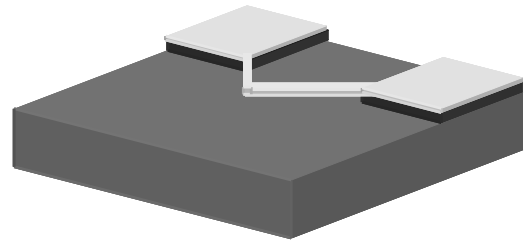

5. $\mathrm{SiO}_{2}$ etching

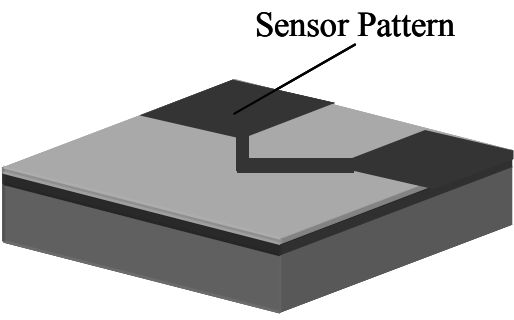

2. EB lithography

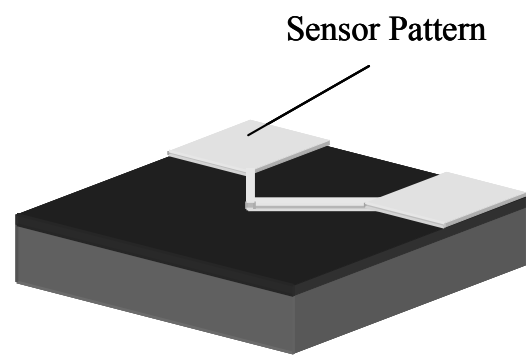

4. Lift off

Suspended Platinum Film

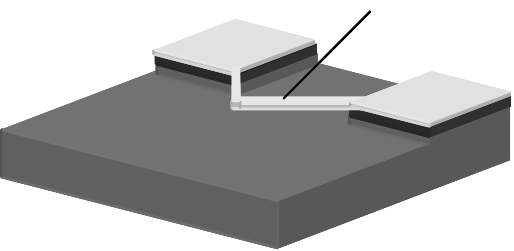

6. Si substrate bulk etching

Fig. 2 Fabrication process of a sensor. Electron beam lithography, the lift-off method for platinum film pattering, and isentropic etching of the underside of the sensor are employed. 


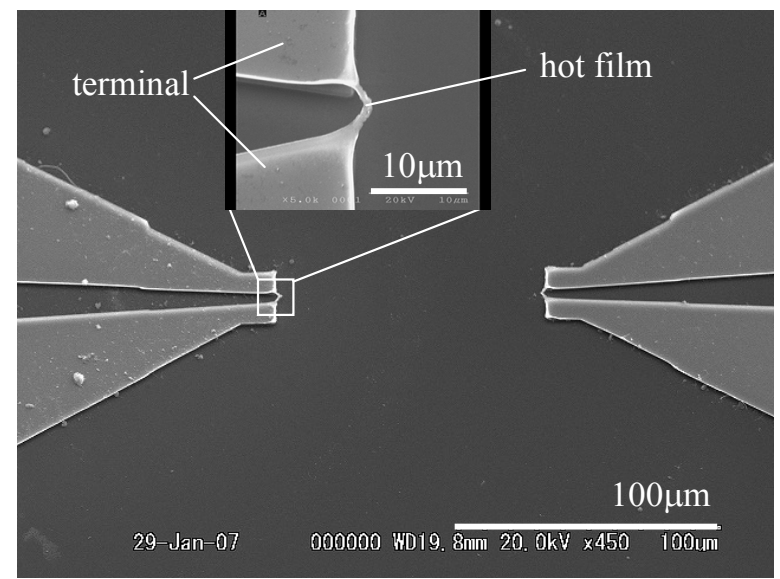

Fig. 3 SEM micrograph of sensors. Two sensors are set face-to-face with a $100-\mu \mathrm{m}$ interval to allow a comparison of the sensing performance. The inset is the closeup of suspended platinum hot film. This confirms complete removal of the material underneath this sensor for thermal insulation.

\subsection{Thermal properties of nanoscale platinum hot-film}

The electrical and thermal properties of this suspended platinum film were measured by the four-wire method from 80 to $300 \mathrm{~K}$ using a liquid nitrogen cryostat (Oxford Instruments, Optistat DN-V). The electrical and thermal conductivities are both less than half the bulk values due to the scale effect. The temperature coefficient of resistance (TCR) is also degraded to less than $0.2 \% /{ }^{\circ} \mathrm{C}$, which is much smaller than the bulk TCR of $0.39 \% /{ }^{\circ} \mathrm{C}$. This is because the electron scattering by the grain boundaries and film interface dominates electron-phonon scattering for thin films. So far, the TCRs of several kinds of films have been determined, for example, $0.13-0.67 \% /{ }^{\circ} \mathrm{C}$ for poly-Si, $0.03 \% /{ }^{\circ} \mathrm{C}$ for $\mathrm{Ni} / \mathrm{Cr}$, and $0.04-0.2 \% /{ }^{\circ} \mathrm{C}$ for CNTs. A higher TCR yields better signals, but stability and linearity are more important. Platinum is chemically stable and has linear TCR. Thus, platinum is recognized as the best sensor material when considering the overall performance in a variety of environments.

\subsection{Alignment of CNTs onto suspended film}

Recently, a synthesis technique that produces vertically aligned CNTs on a localized region has been successfully developed ${ }^{(17)}$. However, this process requires high temperatures that may cause film damage. Consequently, we employed a manipulation method based on dielectrophoresis at room temperature, which is also compatible with batch fabrication ${ }^{(18)}$. Multiwalled CNTs (diameter, 40-60 nm; length, 0.5-2 $\mu \mathrm{m}$; SIGMA ALDRICH, JAPAN) produced by the chemical vapor deposition (CVD) method were used. The thermal conductivity of such commercially available CVD CNTs should be lower than that of single-walled CNTs. Nevertheless, as shown by eq.(4), the thermal conductivity is not a critical determiner of fin performance.

Electrokinetic manipulation techniques for nanotubes have been studies by many researchers ${ }^{(19)-(21)}$. For example, successful results have been reported on the lateral deposition of CNTs between opposing electrodes on a substrate by dielectrophoresis ${ }^{(14),(22),(23)}$. However, the current study requires the CNTs to be oriented perpendicular to the sensor substrate, thus forming ideal pin fins. In order to set the CNTs as close to the normal as possible, we used a 500- $\mu \mathrm{m}$-thick aluminum-deposited glass substrate placed $500 \mu \mathrm{m}$ from the sensor, as shown in Fig. 4. This is intended to make the electric force line align to $45^{\circ}$ from the horizontal. The principle of this technique is explained by Tung et al. considering 


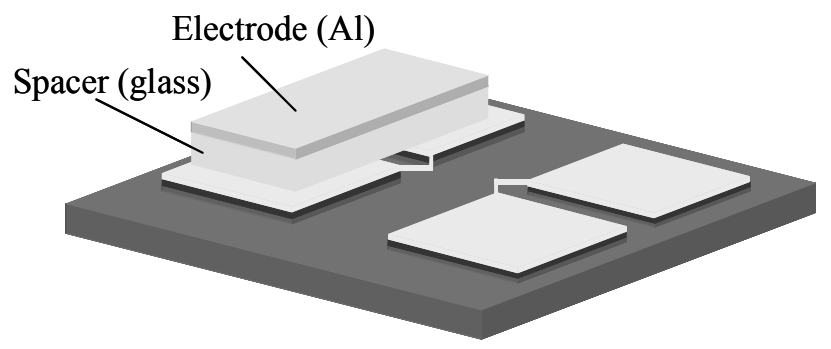

Fig. 4 Experimental setup for dielectrophoresis. The 500- $\mu \mathrm{m}$-thick aluminum-deposited glass substrate is placed $500 \mu \mathrm{m}$ from the sensor

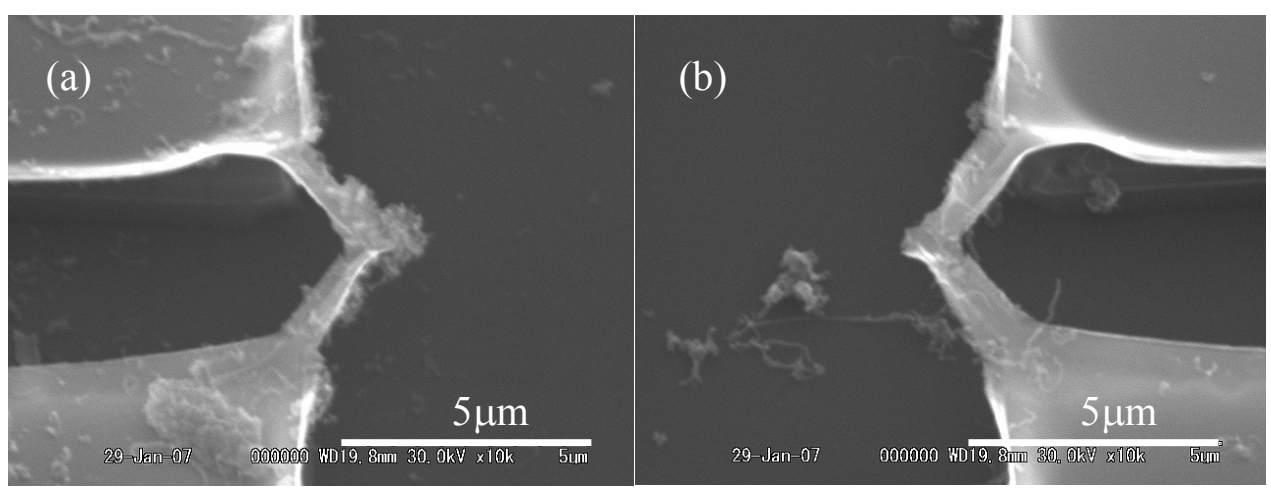

Fig. 5 SEM micrographs of fabricated CNT fins: (a) CNT deposition is localized to the voltage-applied V-shaped sensor. (b) Almost no CNTs are deposited on this sensor.

both dielectrophoresis and Joule-heating effects ${ }^{(12)}$. Normally, the sensor configuration can be straight, however, CNTs are deposited on a variety of places by dielectrophoresis, and we could not concentrically deposit CNTs on a suspended sensor. Because the dielectrophoretic force depends on the gradient of the square of the electric field, we modified our sensor to a V-type configuration, as mentioned above, so as to effectively collect the CNTs on the sensing part.

A CNT suspension was made from $0.1 \mathrm{mg}$ of the above-mentioned CNTs and $100 \mathrm{ml}$ of deionized (DI) water. After dispersion by ultrasonication in a bath for 3 hours, the sensor chip was immersed in the suspension. An AC potential $(10 \mathrm{~V}$ at $10 \mathrm{kHz})$ was applied between one of the sensors and the aluminum electrode for $5 \mathrm{~min}$. After this dielectrophoretic manipulation, the dispersion solution was replaced with ethanol and the chip was dried on a hot plate at $90{ }^{\circ} \mathrm{C}$; this was done to prevent the suspended film from sticking to the substrate. Figure 5 presents an SEM micrograph of a fabricated CNT fin, showing that CNT deposition was successfully localized to the V-shaped sensor (a) on which a potential applied, and almost no deposition occurred on the other sensor (b). In order to make same configuration sensor, we have to fabricate two sensors in one substrate. Therefore, there is possibility of adhesion of CNT due to sedimentation in ethanol dispersion liquid. However, the amount of deposition is different owing to dielectrophoresis. These sensors were tested under the same flow conditions, as is explained in the next subsection.

\subsection{Flow measurement}

The performance of the CNT fins was estimated in the measurement of nitrogen flow in a microchannel made of polydimethylsiloxane (PDMS) by a soft-lithography technique. 


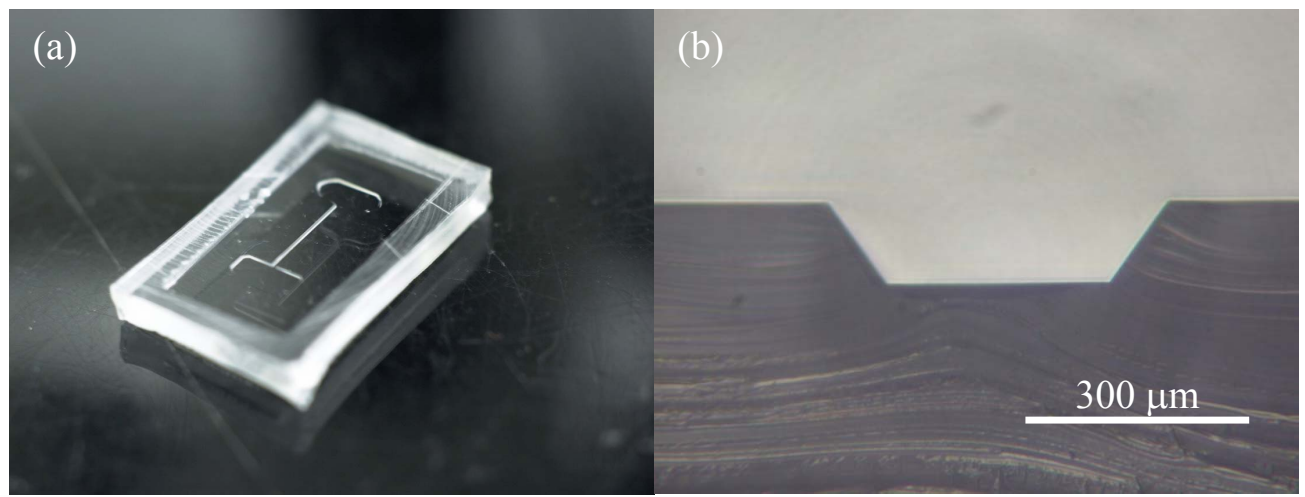

Fig. 6 (a) the PDMS micro channel and (b) the cross section of PDMS micro channel. The channel length is $8 \mathrm{~mm}$. The cross section is trapezoid. The lower base is $300 \mu \mathrm{m}$. The side wall is at an angle of $55^{\circ}$ with the substrate, and then the upper base becomes $440 \mu \mathrm{m}$.

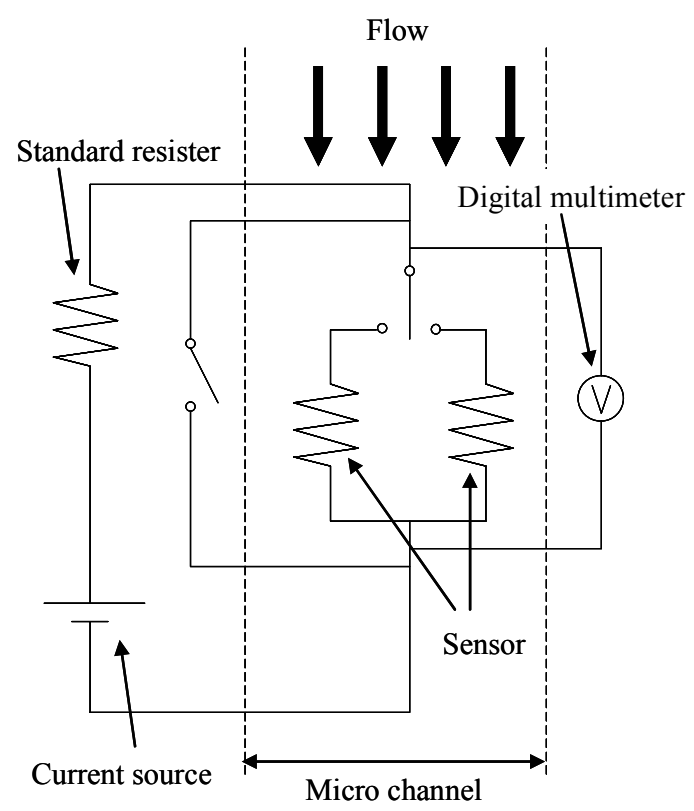

Fig. 7 Experimental setup for flow measurement. The channel is made up of the PDMS. The flow is controlled by mass flow meter.

Figure 6 shows the PDMS microchannel and its cross-section. The PDMS plate had an 8-mm-long groove with a trapezoidal cross-sectional area of $3.7 \times 10^{-8} \mathrm{~m}^{2}$. The sensor chip was set on the PDMS channel. As shown in Fig. 7, the flow conditions at the two sensors were the same. The flow rate was controlled by a mass flow meter and the pressure at the inlet was recorded by a pressure gage. A flow sensor is preferably used at constant temperature (CT) because the signal is dependent on sensor temperature. However, our flow tests were conducted at constant current (CC) because the present study is focused to investigate the feasibility of CNT fins and the suspended film sensor can be destroyed when using the power source designed for commercial hot wire anemometer of CT mode. Here the applied current $I_{0}$ was set to $3 \mathrm{~mA}$, and the obtained voltage $V_{0}$ at no-flow was $916 \mathrm{mV}$ and $924 \mathrm{mV}$ for the no-fin and CNT-fin sensors, respectively. The calculated electrical resistances were $305 \Omega$ and $308 \Omega$, and this difference is due to the non-uniformity in our fabrication process. 


\section{Results and discussion}

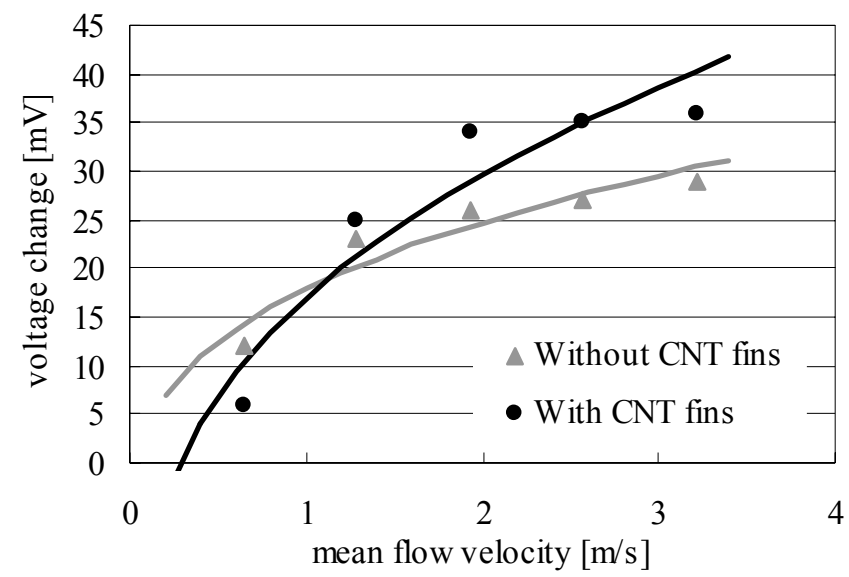

Fig. 8 Measured results with and without CNT fins. The horizontal axis shows mean flow velocity. The vertical axis shows the voltage change by the flow. The black circle is with CNT fins and the gray triangle is without CNT fins. The back line is fitting curve for with CNT fins and the gray line is for without CNT fins.

All of the measured signals, which are voltage changes, of the CNT-fin and no-fin sensors are plotted as black circles and gray triangles in Fig. 8. The fitting curves using the least squared method are also shown. The signals increased with increasing mean flow velocity in micro channel due to heat dissipation from sensor to flow. Because the maximum pressure increase in our experiment was about $0.15 \%$, the effect of pressure change is negligible. The heat dissipation from the CNT-fin sensor was greater than that of no-fin sensor for the mean flow velocity lager than $1.29 \mathrm{~m} \mathrm{~s}^{-1}$. Thus the heat transfer from suspended platinum film to fluid is effectively enhanced by CNTs deposited on it.

Here, a theoretical model represented by the following equation is fitted to the experimental results.

$$
\Delta Q=A \tau_{w}{ }^{1 / 3}+B
$$

where $\Delta Q$ is the heat absorbed by the flow, and $\tau_{w}$ is the shear stress. The shear stress is approximately considered proportional to the mean flow velocity in the channel. Then the voltage change is proportional to the heat dissipation. Therefore, eq. (5) can be rewritten as,

$$
\Delta V=A^{\prime} U^{1 / 3}+B^{\prime}
$$

where $U$ is mean flow velocity. Eq. (5) is usually used in CT mode because the heat transfer from a hot-wire anemometer to the fluid depends on the third power of shear stress at $\mathrm{CT}^{(14)}$. We employ eq. (6) to discuss the characteristics in the present CC mode because an evaluation using the constants $A^{\prime}$ and $B^{\prime}$ is still sufficient for this qualitative investigation. Curve-fitting for the least squared method, which is shown in Fig. 8, affords $A^{\prime}$ ' and $B^{\prime}$ ' of 26.44 and -8.56 for the no fin sensor and 49.15 and -32.20 for the CNT-fin sensor. The larger $A$ ' of the CNT-fin sensor confirms the fundamental advantage of the use of fins in this thermal sensor. In fact, the CNT-fin sensor yields a larger signal when the flow rate is greater than $1.29 \mathrm{~m} \mathrm{~s}^{-1}$.

Comparing obtained $A^{\prime}$ of both sensors, the factor $f$ described in eq. (4) is calculated as about 1.87. Because we used CNTs with an average diameter $D$ of $40 \mathrm{~nm}$ and average length of $1 \mu \mathrm{m}$, the aspect ration $L / D$ becomes 25 . Substituting $f$ and $L / D$ to eq. (4), obtained $c$ is about 0.04 . However, looking at Fig. 5(a), it is expected that the CNT is deposited much more than $c=0.04$. One of the reasons of this discrepancy is the thermal 
contact resistance between CNTs and Pt hot film because their bonding is only due to the weak van der Waals force.

The $B$ ' results require more attention in order to gain an understanding of the workings of the fabricated sensor. The signal obtained from the CNT-fin sensor is lower than that for the no-fin sensor at small flow rates. Our hypothesis explaining this anomaly is as follows: A very small part of the CNT fin should be in contact with the silicon substrate and a small amount of heat dissipation always occurs. This induces no sensitivity at the lower flow velocities, but the greater heat dissipation at higher flow velocities produces an enhanced signal. Above-mentioned discrepancy on $c$ is also explained by this hypothesis.

It should be noted that longer fins are more effective in the laminar flow region because they are exposed to faster-moving fluid. Thus, vertically aligned CNTs should be the most promising design for improved sensitivity. However as shown in Fig. 5, the current CNT was deposited on sensor with aggregates, which causes not only the degradation of heat transfer but the unexpected heat loss into the substrate. In order to make the CNT fin work more effectively, we have to improve the fabrication technique for accomplishing more dispersed and more straight deposition of CNTs.

\section{Conclusion}

We developed a submicroscale flow sensor using a suspended metal film with CNT fins. This senor was made by MEMS/NEMS techniques using the electron beam lithography, the lift-off method, isotropic etching, etc. The use of a suspended film reduces heat loss to substrate, and the CNT fins enhance the heat transfer to the fluid flow. To deposit CNTs on the sensor, we used a manipulation method based on dielectrophoresis. Because the dielectrophoretic force depends on the gradient of the square of the electric field, our sensors have a V-type configuration, CNT deposition was successfully localized to the V-shaped sensor. The advantages of the use of CNT fins were revealed by an experiment on flow measurement using a theoretical model. It can be concluded that by using CNT fins, higher sensitivity, lower power consumption, and a smaller sensing element are possible.

\section{Acknowledgement}

This work was partially supported by Grants-in-Aid for Scientific Research (20360099, 20656140, 21656060). The authors would like to thank Professor Kunihito Nagayama of Kyushu University for fruitful discussions.

\section{References}

(1) Liu, C., Huang, J. B., Zhu, Z., Jiang, F., Tung, S., Tai, Y. C. and Ho, C. M., A Micromashined Flow Shear-Stress Sensor Based on Thermal Transfer Principles, Jornal of Microelectromechanical Systems, Vol. 8, No. 1 (1999), pp. 90-99.

(2) van der Wiel, A. J., Linder, C., de Rooij, N. F. and Bezinge, A., A liquid velocity sensor on the hot-wire principle, Sensors and Actuators A, Vol. 37-38 (1993), pp. 693-697.

(3) Nguyen, N. T. and Kiehnscherf, R., Low-cost silicon sensors for mass flow measurement of liquids and gases, Sensors and Actuators A, Vol. 49 (1995), pp. 17-20.

(4) Go, J. S., Kim, S. J., Lim, G., Yun, H., Lee, J., Song, I. and Pak, Y. E., Heat transfer enhancement using flow-induced vibration of a microfin array, Sensors and Actuators A, Vol. 90 (2001), pp. 232-239.

(5) Kim, T. H., Kim, D. K. and Kim, S. J., Study of the sensitivity of a thermal flow sensor, International Journal of Heat and Mass Transfer, Vol. 52 (2009), pp. 2140-2144.

(6) Szekely, L., Peichert, J. and Freitag, R., Non-invasive nano-flow sensor for application in 
micro-fluidic systems, Sensors and Actuators A, Vol. 133 (2004), pp. 48-53.

(7) Manz, A., Graber, N. and Widmer, H. M., Miniaturized Total Chemical Analysis Systems a Novel Concept for Chwmical Sensing, Sensors and Actuators, B1 (1990), pp. 244-248.

(8) Mailly, F., Giani, A., Bonnot, R., Temple-Boyer, P., Pascal-Delannoy, F., Foucaran, A. and Boyer, A., Anemometer with hot platinum thin film, Sensors and Actuator A, Vol. 94 (2001), pp. 32-38.

(9) Fujii, M., Zhang, X., Xie, H., Ago, H., Takahashi, K., Ikuta, T., Abe, H. and Shimizu, T., Measuring the Thermal Conductivity of a Single Carbon Nanotube, Physical Review Letters, Vol. 95 (2005), 065502.

(10) Frank, S., Poncharal, P., Wang, Z. L. and de Heer, W. A., Carbon nanotube quantum resisters, Science, Vol. 280 (1998), pp. 1744-1746.

(11) Tans, S. J., Verschueren, A. R. M. and Dekker, C., Room-temperature transistor based on a single carbon nanotube, Nature, Vol. 393 (1998), pp. 49-52.

(12) Rueckes, T., Kim, K., Joselevich, E., Tseng, G. Y., Cheung, C. and Lieber, C. M., Carbon nanotube based nonvolatile random access memory for molecular computer, Science, Vol. 289 (2000), pp. 94-97.

(13) Fennimore, A. M., Yuzvinsky, T. D., Han, W. Q., Fuhrer, M. S., Cumings, J. and Zettl, A., Rotational actuators based on carbon nanotubes, Nature, Vol. 424 (2003), pp. 408-410.

(14) Tung, S., Rokadia, H. and Li, W. J., A micro shear stress sensor based on laterally aligned carbon nanotubes, Sensors and Actuator A, Vol. 133 (2007), pp. 431-438.

(15) Incropera, F. P. and DeWitt, D. P., Fundamentals of heat and mass transfer, fifth ed., (2002), p. 132, John Wiley \& Sons.

(16) Launay, S., Fedorov, A. G., Joshi, Y., Cao, A. and Ajayan, P. M., Hybrid micro-nano structured thermal interfaces for pool boiling heat transfer enhancement, Microelectronics Journal, Vol. 37 (2006), pp. 1158-1164.

(17) Murakami, Y., Chiashi, S., Miyauchi, Y., Hu, M., Ogura, M., Okubo, T. and Maruyama, S., Growth of vertically aligned single-walled carbon nanotube films on quartz substrates and their optical anisotropy, Chemical Physics Letters, Vol. 385 (2004), pp. 298-303.

(18) Ramos, A., Morgan, H., Green, N. G. and Castellanos, A., Ac electrokinetics: a review of forces in microelectrode structures, Journal of Physics D: Applied Physics, Vol. 31 (1998), pp. 2338-2353.

(19) Yamamoto, K., Akita, S. and Nakayama, Y., Orientation of Carbon Nanotubes Using Electrophoresis, Japanese Journal of Applied Physics, Vol. 35 (1996), pp. 917-918.

(20) Suehiro, J., Zhou, G. and Hara, M., Detection of partial discharge in SF6 gas using a carbon nanotube-based gas sensor, Sensors and Actuators B, Vol. 105 (2005), pp. 164-169.

(21) Suehiro, J., Zhou, G. and Hara, M., Fabrication of a carbon nanotube-based gas sensor using dielectrophoresis and its application for ammonia detection by impedance spectroscopy, Journal of Physics D: Applied Physics, Vol. 36 (2003), pp. 109-114.

(22) Fung, C. K. M., Wong, V. T. S., Chan, R. H. M. and Li, W. J., Dielectrophoretic Batch Fabrication of Bundled Carbon Nanotube Thermal Sensors, IEEE Transactions on Nanotechnology, Vol.3, No. 3 (2004), pp. 395-403.

(23) Seo, H. W., Han, C. S., Choi, D. G., Kim, K. S. and Lee, Y. H., Controlled assembly of single SWNTs bundle using dielectrophoresis, Microelectronic Engineering, Vol. 81 (2005), pp. 83-89. 